\title{
Neumonía en el anciano. Pacientes residentes en instituciones cerradas y clases de riesgo
}

\author{
L. DE OÑA LACASTA, M. GÓMEZ FERNÁNDEZ, J. CELDRÁN GIL \\ Unidad de Neumología. Hospital Nuestra Señora del Prado. Talavera de la Reina. Toledo
}

\author{
PNEUMONIA IN THE EDERLY. NURSING HOME PATIENTS AND \\ RISK CLASSES
}

\section{RESUMEN}

La residencia del paciente y enfermedades subyacentes pueden incrementar la gravedad y el riesgo de la neumonía en el paciente anciano.

Objetivos: Analizar las características demográficas y la mortalidad de los pacientes ancianos ingresados con neumonía, según su procedencia y determinar la distribución por clases de riesgo.

Metodo: Se estudiaron los pacientes de al menos 65 años, ingresados con neumonía durante 12 meses, recogiendo datos demográficos, clínicos y de las enfermedades coexistentes, asignando cada paciente a una clase de riesgo (de II a V).

Resultados: Se estudiaron 103 pacientes (edad media de 78,5 añosDE 7,16-), 67 hombres y 36 mujeres, el $80 \%$ pertenecientes a clases de riesgo alto, con una mortalidad del $15,5 \%$. El 17,5\% procedían de una Residencia asistida, con una edad superior al resto (edad media de 82,7 años-DE 5,72-) ( $\mathrm{p}<0,002)$, mayor proporción de mujeres y de pacientes de clases de riesgo alto, y una menor estancia hospitalaria.

Conclusiones: Los pacientes ancianos con neumonía tienen riesgo alto. Los procedentes de residencia son de mayor edad, mayor riesgo, mayoritariamente mujeres, y tenían menos estancia hospitalaria.

PALABRAS CLAVE: Neumonía. Riesgo. Anciano. Residencia. Demencia.

De Oña Lacasta L, Gómez Fernández M, Celdrán Gil J. Neumonía en el anciano. Pacientes residentes en instituciones cerradas y clases de riesgo. An Med Interna (Madrid) 2002; 19: 115-119.

\section{INTRODUCCIÓN}

La neumonía adquirida en la comunidad (NAC) tiene una importante prevalencia y un impacto socioeconómico elevado (1). Las sociedades científicas hacen constantes esfuerzos por unificar criterios diagnósticos y terapéuticos (2). La neumonía en los pacientes ancianos puede tener una presentación clínica diferente a la de otros grupos de edad (3). La confusión, las alteraciones de la capacidad funcional o la descompensación de las enfermedades subyacentes pueden ser las únicas manifestaciones de la NAC $(3,4,5)$.

\begin{abstract}
The residence and underlying diseases could be increase the severity and risk in the pneumonia in the elderly patient.

Background: To analyse demographics qualities and mortality in elderly inpatients with pneumonia, in relation with kind of residence, and to determine the risk classes distribution.

Method: Were studied inpatients with 65 years old or more, with pneumonia in a period of 12 months, taking demographics values and underlying diseases, including each patient in a risk class (II to V).

Results: One hundred and three patients were studied (78,5 years mean age-SD 7,16), 67 males and 36 females, $80 \%$ includes in high risk classes, with $15,5 \%$ mortality. $17,5 \%$ were home nursing, older $(82,7$ years mean age-SD 5,72-) ( $p<0,002)$, predominantly females and in high risk classes, and with lower length of hospital stay.

Conclusions: The elderly patients with community-acquired pneumo nia was high risk. Home nursing was older, more risk and lower length of hospital stay.
\end{abstract}

KEY WORDS: Pneumonia. Risk. Elderly. Home nursing. Dementia. 
Variables como la frecuencia respiratoria (FR) de 30 r.p.m. o más, Tensión Arterial Diastólica de $60 \mathrm{mmHg}$ o menos y la confusión mental pueden identificar más ajustadamente los pacientes con riesgo de muerte (5).

Por otra parte, todavía existen controversias sobre la necesidad de incluir o no a los pacientes con Neumonía adquirida en la residencia dentro de los pacientes con NAC. Este tipo de pacientes presentan un patrón etiológico más similar al de la neumonía nosocomial, mayor proporción de aspiraciones, más mortalidad, así como asocian más frecuentemente accidentes cerebrovasculares y demencia $(7,8)$.

Todos estos datos publicados, tratan de valorar el riesgo de muerte de los pacientes. Sin embargo, recientemente se ha propuesto un sistema de valoración del riesgo más global y que permite encuadrar a cada paciente, de forma individual, dentro de una clase de riesgo, permitiendo conocer más exactamente el riesgo de dicho paciente. Esto autores han basado dicho sistema, entre otras variables, en la edad, el sexo, la presencia de comorbilidad, constantes vitales anómalas y alteraciones analíticas y radiológicas, todas las cuales permiten una estimación del riesgo de muerte causada por neumonía (9).

Conocer las características demográficas, la distribución de los grupos de riesgo así como la comorbilidad y mortalidad existente, puede permitir un mejor manejo de la NAC en el anciano. Las diferencias existentes en la clínica, evolución y pronóstico de la NAC en los pacientes ancianos, respecto a otros grupos de edad, hacen necesario una mejor comprensión de esta patología.

No hemos encontrado en la bibliografía trabajos realizados en nuestro medio que hallan estudiado las características de los pacientes ancianos ingresados con NAC, ni si existen diferencias en aquellos casos procedentes de residencias asistidas, en función de las clases de riesgo de los mismos.

Nuestro objetivo era analizar las características demográficas, clínicas y la mortalidad de los pacientes de 65 años o más ingresados por NAC, teniendo en cuenta su residencia habitual previa al ingreso. Queríamos determinar también la distribución por clases de riesgo de dichos pacientes y observar las diferencias que pudieran existir.

\section{MATERIAL Y MÉTODOS}

Se recogieron consecutivamente para el estudio pacientes adultos de 65 años de edad o más diagnosticados de NAC, ingresados en una unidad de Neumología durante un período de doce meses. La Unidad de Neumología de nuestro centro recibe la mayor parte de los pacientes ingresados en el hospital por NAC, independientemente de la edad o la patología subyacente. El diagnóstico de NAC se basó en la presencia de un infiltrado nuevo en la radiografía de tórax, síntomas indicativos de infección del tracto respiratorio inferior o sintomatología clínica que la hiciese sospechar y sin hospitalización previa en el mes anterior. Se excluyeron del estudio aquellos pacientes cuyo diagnóstico definitivo no fue el de NAC. Para el diagnóstico de NAC se consideró el criterio del facultativo que cursó el ingreso así como el del neumólogo que siguió al paciente ingresado y procedió después al alta y seguimiento en las consultas del hospital.

Se recogieron de los pacientes durante el ingreso los siguientes datos: edad, sexo, residencia, comorbilidad, cons- tantes vitales como FR, frecuencia cardíaca (FC), tensión arterial (TA), temperatura $\left(\mathrm{T}^{\mathrm{a}}\right)$ y estado mental, así como datos analíticos (leucocitos, proteinemia, $\mathrm{PH}$ arterial, $\mathrm{PaO}_{2}$ arterial, nitrógeno uréico, natremia, glucemia y hematocrito) y radiológicos. Igualmente se recogieron datos sobre posibles episodios aspirativos, falta de respuesta al tratamiento previo o problemas sociales para su cumplimiento, el tratamiento antibiótico empírico inicial con el que se ingresó al paciente y la duración de la estancia hospitalaria.

Mediante los datos obtenidos se procedió a la asignación de cada paciente a una determinada clase de riesgo. Para ello se utilizó como escala de puntuación los valores que se observan en el anexo 1, que permitían distribuir a los pacientes en clases de riesgo, de la II a la $\mathrm{V}$, según el menor o mayor riesgo (9), teniendo en cuenta que la clase de riesgo I requiere menos de 50 años y no se incluye en el estudio.

Para el análisis estadístico se utilizó un ordenador personal y se realizó mediante la determinación de proporciones y su comparación mediante la Prueba z para grupos independien-

\begin{tabular}{ll} 
& ANEXO 1 \\
CARACTERÍSTICAS & PUNTOS ASIGNADOS \\
\hline $\begin{array}{c}\text { Factor demográfico } \\
\text { Edad: Hombres } \\
\text { M ujeres }\end{array}$ & Edad en años \\
& Edad en años - 10
\end{tabular}

Vivienda habitual en residencia asistida

$+10$

Enfermedad coexistente

Enfermedad neoplásica $\quad+30$

Enfermedad hepática $\quad+20$

Insuficiencia cardíaca $\quad+10$

Enfermedad cerebrovascular $\quad+10$

Enfermedad renal +10

Hallazgos en la exploración física

Alteración del estado mental $\quad+20$

FR igual o superior a $30 \mathrm{rpm} \quad+20$

PAS menor a $90 \mathrm{mmHg}+20$

Temperatura $<35 \stackrel{\circ}{\circ} 0$

igual $0>40 \stackrel{\circ}{C}+15$

FC igual o $>125$ lpm $\quad+10$

Hallazgos analíticos y radiológicos

$\mathrm{PH}$ arterial $<7,35+30$

$\mathrm{N}$ itrógeno uréico (BUN) igual

$0>30 \mathrm{mg} / \mathrm{dl}+20$

Sodio $<130 \mathrm{mmol} / \mathrm{L} \quad+20$

Glucemia igual $0>250 \mathrm{mg} / \mathrm{dl}+10$

Hematocrito $<30 \% \quad+10$

Presión parcial arterial de oxígeno

$\begin{array}{ll}<60 \mathrm{mmHg} & +10 \\ \text { Derrame pleural } & +10\end{array}$

M odificado de Fine y cols. 9 . 
tes, así como la estimación de medias y su comparación mediante la Prueba t, corregida para muestras pequeñas. Se consideró estadísticamente significativo un valor de $\mathrm{p}<0,05$.

\section{RESULTADOS}

Se incluyeron durante el período de estudio 103 pacientes diagnosticados de NAC, con una edad media de 78,5 años (DE 7,16). Cerca del 70\% de ellos tenía 75 años o más. Fallecieron durante el ingreso 16 pacientes del total $(15,5 \%)$, y el 19,5\% (14 pacientes) si tenemos en cuenta sólo a los pacientes de al menos 75 años (Tabla I). Sin embargo, no se observaron diferencias significativas respecto a la edad entre los pacientes que fallecieron y los que sobrevivieron. Existía una mayor proporción de hombres que de mujeres entre los pacientes supervivientes $(56 / 31)$, que todavía era mayor entre los pacientes que fallecieron (11/5), aunque estas diferencias no fueron significativas (Tabla I).

Los pacientes ingresados estaban distribuidos en las distintas clases de riesgo, según se observa en la Tabla II, teniendo en cuenta que, por definición, no podían existir pacientes pertenecientes a la clase de riesgo I. Podemos observar que más del $80 \%$ pertenecían a las clases de riesgo más alto (IV y $\mathrm{V})$. Es en estas clases de riesgo donde se produce la mortalidad, existiendo diferencias significativas en este sentido entre la clase V $(33,3 \%)$ y la clase IV $(4,7 \%)$ (p<0,0007). Existía una mayor proporción de hombres que de mujeres (67/36) en la totalidad de nuestra muestra, así como en las diferentes cla- ses de riesgo, sin diferencias significativas entre ellas (Tabla II).

Del total de pacientes ingresados por NAC, 18 (17,5\%) procedían de una Residencia asistida, donde habían residido al menos un mes (Tabla I). La proporción era mayor entre los pacientes que habían fallecido $(31,25 \%)$ que entre los supervivientes $(15 \%)$, aunque estas diferencias no eran significativas $(\mathrm{p}=0,058)$ (Tabla I). La edad de los pacientes que procedían de una residencia era de 82,7 años (DE 5,72), significativamente mayor que el resto $(77,6$ años - DE 7,14-) $(\mathrm{p}<0,002)$ (Tabla III). Se observaba un predominio de hombres en el grupo de pacientes procedentes de domicilio familiar (59/26), mientras que en el grupo de pacientes procedentes de residencia eran más frecuentes las mujeres $(8 / 10)(\mathrm{p}<0,022)$ (Tabla III). No existían diferencias significativas respecto a la estancia hospitalaria entre el grupo de pacientes procedentes de residencia y de domicilio familiar (Tabla III). Sin embargo, sí observamos diferencias en la mortalidad entre los pacientes procedentes de residencia, donde era mayor, y el resto de pacientes (Tabla III).

Cuando se analiza la distribución de estos pacientes procedentes de residencia asistida por clases de riesgo observamos que, de todos ellos, 14 pacientes $(77,8 \%)$ pertenecían a la clase V y 4 pacientes $(22,2 \%)$ a la clase IV. Esto significa que hay una mayor proporción de pacientes que procedían de una residencia incluidos en la clase $\mathrm{V}(33,3 \%)$ que los incluidos en la clase IV $(9,3 \%)(\mathrm{p}<0,0035)$. Igualmente, encontramos que había mayor proporción de pacientes procedentes de residencia asignados a la clase de riesgo V (14 de 18) que en el

TABLA I

CARACTERÍSTICAS DEM O GRÁFICAS, PRO CEDENCIA Y DETERIORO CO GNITIVO DE LOS PACIENTES ANCIANOS INGRESADOS POR NAC

\begin{tabular}{lcccccc}
\hline & № & Edad (DE) & $\begin{array}{c}\text { № pacientes } \\
75 \text { años (\%) }\end{array}$ & Sexo (H/M) & $\begin{array}{c}\text { Procedentes de } \\
\text { Residencia(\%) }\end{array}$ & $\begin{array}{c}\text { Pacientes con } \\
\text { Demencia }(\%)\end{array}$ \\
\hline Supervivientes & 87 & $81,8(7,07)$ & $54(66,7 \%)$ & $56 / 31$ & $13(15)$ & $13(15)$ \\
No supervivientes & 16 & $77,8(6,92)$ & $14(87,5 \%)$ & $11 / 5$ & $5(31,25)$ & $3(23)$ \\
Total & 103 & $78,5(7,16)$ & $72(69,9 \%)$ & $67 / 36$ & $18(17,5)$ & $16(15,5)$ \\
\hline
\end{tabular}

DE: desviación estándar. H/M : hombres/mujeres.

TABLA II

DISTRIBU CIÓ N POR CLASES DE RIESGO Y DATOS DEM O GRÁFICOS DE LOS PACIENTES ANCIANOS CON NAC

\begin{tabular}{lcccc}
\hline Clase de riesgo & № $(\%)$ & № No SV $(\%)$ & Sexo $(H / M)$ & EM $(\mathrm{DE})$ \\
\hline II $(<70)$ & $3(2,9)$ & 0 & $3 / 0$ & $68(1,73)$ \\
III $(71-90)$ & $15(14,6)$ & 0 & $8 / 7$ & $74,5(5,48)$ \\
IV $(91-130)$ & $43(41,7)$ & $2(4,7)^{*}$ & $24 / 19$ & $78(7,24)$ \\
V $(>130)$ & $42(40,8)$ & $14(33,3)^{*}$ & $32 / 10$ & $81(6,56)$ \\
Total & 103 & $16(15,5)$ & $67 / 36$ & $78,5(7,16)$ \\
\hline
\end{tabular}

No SV: no supervivencia; EM : Edad M edia; ${ }^{*} p<0,0007$; DE: desviación estándar. 


\section{TABLA III}

DATOS DEM O GRÁFICOS, ESTANCIA Y M ORTALIDAD HOSPITALARIA EN LOS PACIENTES CON NAC PRO CEDENTES DE RESIDENCIA ASISTIDA

\begin{tabular}{lccc}
\hline & $\begin{array}{c}\text { Procedentes de } \\
\text { residencia }\end{array}$ & $\begin{array}{c}\text { Resto de } \\
\text { pacientes }\end{array}$ & Significación \\
\hline $\begin{array}{l}\text { № } \\
\text { Edad (años) } \\
\text { (DE) }\end{array}$ & 18 & 85 & \\
$\begin{array}{l}\text { Sexo (H/M) } \\
\text { Estancia SV } \\
\text { (días) (DE) }\end{array}$ & $82,7(5,72)$ & $77,6(7,14)$ & $\mathrm{P}<0,002$ \\
$\begin{array}{l}\text { M ortalidad } \\
\text { № (\%) }\end{array}$ & $5(27,8)$ & $59 / 26$ & $\mathrm{P}<0,022$ \\
$\begin{array}{l}\text { Asignados } \\
\text { a clase V (\%) }\end{array}$ & $14(77,8)$ & $8(5,8)$ & $\mathrm{NO}$ \\
\hline
\end{tabular}

SV: Supervivientes. DE: desviación estándar. H/M : hombres/mujeres.

resto de pacientes (28 de 85), que procedían del domicilio familiar $(\mathrm{p}<0,0007)$ (Tabla IV). Dado que proceder de una residencia es uno de los factores que se tienen en cuenta en la puntuación para asignar a las clases de riesgo, se descontó dicha puntuación, y se asignaron de nuevo las clases de riesgo, no observándose diferencias en estas proporciones. No se observaba diferencia en la edad entre los pacientes procedentes de una residencia asistida incluidos en la clase de riesgo $\mathrm{V}$ (82,3 años- DE 5,6-) y el resto de pacientes de esa clase (80,4 años -DE 7-) (Tabla IV). Tampoco se observó diferencia en la clase IV. Respecto a la distribución por sexos, en la clase V los pacientes procedentes de residencia se distribuyen al $50 \%$

\section{TABLA IV}

DATOS DEM O GRÁFICOS, ESTANCIA Y M ORTALIDAD HOSPITALARIA EN LOS PACIENTES CON NAC PRO CEDENTES DE RESIDENCIA ASIGNADOS DE CLASE DE RIESGO V

\begin{tabular}{lccc}
\hline & $\begin{array}{c}\text { Procedentes de } \\
\text { residencia }\end{array}$ & $\begin{array}{c}\text { Resto de } \\
\text { pacientes }\end{array}$ & Significación \\
\hline $\begin{array}{l}\text { No } \\
\text { Edad (años) } \\
\text { (DE) }\end{array}$ & 14 & 28 & \\
Sexo (H/M) & $82,3(5,6)$ & $80,4(7)$ & No \\
$\begin{array}{c}\text { Estancia SV } \\
\text { (días) (DE) }\end{array}$ & $9,11(4,86)$ & $12,32(8,39)$ & $\mathrm{p}<0,02$ \\
$\begin{array}{c}\text { Mortalidad } \\
\text { No (\%) }\end{array}$ & $5(35,7)$ & $9(32,1)$ & No \\
\hline
\end{tabular}

SV: Supervivientes. DE: desviación estándar. H/M : hombres/mujeres. entre hombres y mujeres, mientras que predominan los hombres en el grupo de pacientes que no procede de residencia $(\mathrm{p}<0,003)$ (Tabla IV). La mortalidad es similar entre los dos grupos de esta clase $\mathrm{V}$, procedentes de residencia $(35,7 \%)$ y de domicilio familiar $(32,1 \%)$. Sí se observó una menor estancia hospitalaria entre los pacientes procedentes de residencia (9,11 días -DE4,86-) que entre los procedentes de domicilio familiar (12,32 -DE 8,39-) (p<0,02) (Tabla IV).

\section{DISCUSIÓN}

La mortalidad del grupo de pacientes ancianos es acorde con lo descrito en la bibliografía $(5,10)$. Existe un predominio de hombres, siendo esta proporción mayor entre los fallecidos. Nuestros pacientes ancianos con neumonía se encuentran distribuidos en las clases de riesgo más alto (IV y V), en mayor proporción que algunos datos publicados recientemente, aunque con una mortalidad en dichas clases similar a las encontradas por esos autores (5).

Los pacientes de nuestra muestra presentaban una edad media muy similar a estudios recientes en poblaciones de estas características (5). Sin embargo estaba compuesta por un elevado número de pacientes de 75 años o más (grandes ancianos), en mayor proporción que la encontrada por estos autores. Existen controversias respecto a que la edad sea un factor independiente de riesgo de muerte en los pacientes con neumonía, y encontramos estudios que observan mayor riesgo en los pacientes con mayor edad $(11,12)$, y otros que no lo observan (6). Existe la posibilidad de que lo que determine una mayor mortalidad sea el agrupamiento de factores de riesgo en un determinado paciente, y hay indicios de que lo que determina el riesgo de muerte es la situación general del paciente (9), en la cual la edad es un dato más, pero no el determinante. Nosotros hemos observado en nuestra muestra que los pacientes que fallecen no tienen una edad significativamente superior.

La proporción de pacientes procedentes de residencia está en consonancia con otras series (5). Estos pacientes, en nuestra serie, tienen una edad mayor que el resto del grupo, y superior a la de algunos otros estudios (8), aunque respecto a este dato hay que tener en cuenta que casi tres cuartas partes de todos nuestros pacientes eran grandes ancianos (75 años o más), lo que constituye un elevado porcentaje, como ya se ha comentado.

En nuestro estudio, aunque las diferencias no son significativas, existe una tendencia a una mayor proporción de pacientes procedentes de residencia entre los fallecidos. Es conocido que los pacientes procedentes de residencias cerradas utilizan con más frecuencia los servicios médicos, son más hospitalizados y presentan más frecuentemente infecciones, especialmente las respiratorias $(13,14)$. Igualmente, la mortalidad entre los residentes de nuestra muestra era superior a la del resto de pacientes. La mortalidad en ambos grupos, residentes y resto de pacientes, es similar a la presentada en recientes estudios (7). Esto, unido al hecho de que existe una mayor proporción de pacientes pertenecientes a la clase $\mathrm{V}$ entre los residentes que entre el resto de pacientes, sugiere que los pacientes procedentes de residencia son de mayor gravedad y, por tanto, mayor riesgo, además de mayor edad. Este mayor riesgo viene reafirmado por el dato de que la mortalidad dentro de la clase de riesgo $\mathrm{V}$ es, sin embargo, similar entre los residentes y el resto de pacientes, de manera que la 
mayor mortalidad en los pacientes procedentes de residencia parece venir dada por presentar este tipo de pacientes neumonías más graves, ya que a igualdad de riesgo hay en cambio igual mortalidad.

El grupo total de pacientes procedentes de instituciones cerradas no presentaban diferencias con los pacientes procedentes del domicilio familiar respecto a la estancia hospitalaria. Sin embargo, cuando se analiza por clases de riesgo, existía una menor estancia hospitalaria en los pacientes de clase $\mathrm{V}$ que procedían de una residencia en comparación con el resto de pacientes de esa clase, lo que puede explicarse por el apoyo $\mathrm{y}$ control tras el alta que los pacientes que son remitidos a una residencia asistida pueden tener. Al igual que nosotros, otros autores han encontrado una menor estancia hospitalaria en el grupo de pacientes con neumonía procedentes de residencia (8). Esto debe hacer recapacitar sobre el beneficio de un adecuado control tras el alta hospitalaria en los pacientes ancianos, especialmente en situaciones de gravedad.

Respecto al sexo de los pacientes, existía un ligero predominio de mujeres en los residentes en comparación con el resto de pacientes, donde predominaban los hombres de forma destacada. Existen trabajos en nuestro medio con pacientes ancianos con NAC donde, como en nuestro caso, la proporción general de varones es mayor (3). También hemos encontrado datos en la literatura donde, en grandes series, hay una proporción similar entre hombres y mujeres en los pacientes procedentes de residencia (8), datos muy similares a los nuestros.

Es de destacar la alta proporción de pacientes procedentes de una residencia que existía en la clase de riesgo más alto, así como la elevada proporción que estos pacientes suponían en la citada clase de riesgo. Esto nos sugiere que estos pacientes presentaban neumonías de mayor gravedad cuando acudían al hospital. Sin embargo, la edad dentro de esta clase de riesgo, así como la mortalidad, era similar a la del resto de los pacientes, sugiriendo todo ello que los pacientes que llegaron al hospital, procedentes de una residencia estaban más graves que el resto y que probablemente por ello fallecían en mayor proporción. En la literatura analizada no hemos encontrado un análisis semejante con este subgrupo de pacientes con neumonía, y dado el diseño de nuestro estudio no es posible sacar conclusiones definitivas, siendo necesario por ello la realización de más estudios. No obstante, nuestros datos orientan a que los pacientes que ingresan en una planta con el diagnóstico de neumonía y que proceden de una residencia, deben ser vistos como pacientes con neumonías más graves y de mayor riesgo de muerte por ello. Todo ello nos induce a pensar que cuanto más discriminemos las variables de riesgo de cada paciente, entre ellas la residencia habitual, mejor podremos realizar su abordaje. Pensamos, como otros autores, que la edad por sí misma no es un criterio para no utilizar medidas agresivas en el tratamiento de la NAC (15).

En conclusión, los pacientes con neumonía que proceden de una residencia son pacientes pertenecientes a clases de mayor riesgo y, probablemente por ello, de mayor mortalidad, son pacientes con mayor edad y con similar distribución entre ambos sexos. Estos pacientes tienen, para un riesgo similar, una menor estancia hospitalaria, lo que puede deberse al apoyo que tras el alta puede proporcionar el centro asistido.

\section{Bibliografía}

1. Garibaldi RA. Epidemiology of community acquired respiratory tract infections in adults: incidence, etiology and impact. Am J Med 1985; 78: 32-7.

2. American Thoracic Society. Guidelines for the initial management of adults with community-acquired pneumonia: diagnosis, assessment of severity, and initial antimicrobial therapy. Am Rev Respir Dis 1993; 148: 1418-26.

3. González Quijada S, Galdós Barroso M, Riego Valledor A, Fernández Alvarez G, Galindo Vicente MC. Neumonía en el anciano: ¿es atípica su presentación clínica? An Med Interna (Madrid) 2001; 18: 124-6.

4. Riquelme R, Torres A, el-Ebiary M, Mensa J, Estruch R, Ruiz M, et al. Community-acquired pneumonia in the elderly. Clinical and nutritional aspects. Am J Respir Crit Care Med 1997; 156 (6): 1908-14.

5. Ewig S, Kleinfeld T, Bauer T, Seifert K, Schafer H, Goke N. Comparative validation of prognostic rules for community-acquired pneumonia in an elderly population. Eur Respir J 1999; 14 (2): 370-5.

6. Riquelme R, Torres A, el-Ebiary M, Puig de la Bellacasa J, Estruch R, Mensa $\mathrm{J}$ et al. Community-acquired pneumonia in the elderly. A multivariate analysis of risk and prognostic factors. Am J Respir Crit Care Med 1996; 154: 1450-5.

7. Marrie TJ, Blanchard W. A comparison of nursing home-acquired pneumonia patients with patients with community-acquired pneumonia and nursing home patients without pneumonia. J Am Geriatr Soc 1997; 45: 50-5.

8. Marrie TJ, Durant H, Kwan C. Nursing home-acquired pneumonia. A case-control study. J Am Geriatr Soc 1986; 34: 697-702.

9. Fine MJ, Auble ThE, Yealy DM, Hanusa BH, Weissfeld LA, Singer $\mathrm{DE}$, et al. A prediction rule to identify low-risk patients with community-acquired pneumonia. N Engl J Med 1997; 336: 243-50.

10. Venkatesan P, Gladman J, Macfarlane JT, Barer D, Berman P, Kinnear $\mathrm{W}$, et al. A hospital study of community acquired pneumonia in the elderly. Thorax 1990; 45 (4): 254-8.

11. Farr BM, Woodhead MA, Macfarlane JT, Barlett CL, McCraken JS, Wadswoth J, et al. Risk factors for community-acquired diagnosed by general practitioners in the community. Respir Med 2000; 94 (5): 422 -

12. Lim WS, Lewis S, Macfarlane JT. Severity prediction rules in community acquired pneumonia: a validation study. Thorax 2000; 55: 219-23.

13. Garibaldi RA, Brodine S, Matsumiya S. Infections among patients in nursing homes: policies, prevalence, problems. N Engl J Med 1981 (Sep) 24; 305 (13): 731-5.

14. Irvine PW, Van Buren N, Crossley K. Causes for hospitalization of nursing home residents: the role of infection. J Am Geriatr Soc $1984 ; 32$ (2): 103-7.

15. Brancati FL, Chow JW, Wagener MM, Vacarello SJ, Yu VL. Is pneumonia really the old man's friend? Two-year prognosis after community-acquired pneumonia. Lancet 1993; 342: 30-3. 